\title{
THE NSW HEALTH RESOURCE DISTRIBUTION FORMULA AND HEALTH INEQUALITIES
}

Andrew Gibbs, Rick Sondalini, Jim Pearse

Funding and Systems Policy Branch

NSW Department of Health

\section{BACKGROUND}

A key feature of the NSW health system is its 17 geographically-based area health services (AHSs). Funding to the AHSs by the NSW Department of Health has been guided by the objective of providing the AHSs with a share of resources that allows the achievement of comparable access to health services, assuming the achievement of reasonable levels of efficiency. ${ }^{1}$ The mechanism for achieving this objective is the Resource Distribution Formula (RDF). Since the late 1980s, the formula has been used to guide the allocation of funding to the AHSs and to monitor progress towards the achievement of geographical equity in health funding across NSW.

The RDF reflects a strong commitment to the idea that population-based funding should be directed to communities in accordance with their health needs, thus addressing one potential contributor to health inequalities: inequitable access to health services. It has been suggested that a population needs-based funding approach would also address equity at a national level, through better integration and targeting of various funding streams based on need. ${ }^{2}$
This paper briefly describes the RDF and discusses the role the formula might play in reducing health inequalities and responding to the inequitable distribution of health needs across the NSW population.

\section{DESCRIPTION OF THE RESOURCE DISTRIBUTION FORMULA}

The RDF is constructed using two sets of measures: measures that attempt to measure the relative need of populations within the AHSs, and measures that attempt to address legitimate differences in service delivery costs between the AHSs. These measures are considered in relation to each of the major programs of the NSW health system.

The starting point for need-measures is typically the population of each area, both current estimates and future projections. Consideration is then given to the influence of the age and sex composition on the need for services. Finally, attention is paid to other factors that are demonstrated to influence the need for services. In this context, the NSW Department of Health has developed, in collaboration with the Health Services Research Group at the University of Newcastle, a 'health needs index' for non-tertiary and non-obstetrics services. The development of this index parallels research sponsored by the English National Health Service for the development of indices of need for use in their funding arrangements. ${ }^{3,4,5}$

\begin{tabular}{|c|c|c|c|c|}
\hline \multicolumn{5}{|c|}{ NSW HEALTH NEEDS INDEX BY AREA HEALTH SERVICE ${ }^{6}$} \\
\hline Health Area & $\begin{array}{l}\text { SMR } \\
(90-92)\end{array}$ & $\begin{array}{r}\text { EDOCC } \\
(1991)\end{array}$ & $\begin{array}{c}\text { Rurality } \\
\text { Index }\end{array}$ & Need Index \\
\hline Northern Sydney & 75.1 & 112.9 & 16.6 & 82.5 \\
\hline South Eastern Sydney & 97.0 & 105.8 & 16.7 & 93.9 \\
\hline Wentworth & 98.3 & 101.5 & 15.1 & 97.7 \\
\hline Western Sydney & 103.3 & 100.0 & 16.1 & 99.4 \\
\hline Illawarra & 98.8 & 96.5 & 14.6 & 100.2 \\
\hline South West Sydney & 101.2 & 95.3 & 15.3 & 101.1 \\
\hline Central Coast & 102.1 & 95.8 & 13.6 & 102.9 \\
\hline Central Sydney & 115.4 & 102.1 & 17.0 & 102.9 \\
\hline Hunter & 104.2 & 95.6 & 14.3 & 103.2 \\
\hline Northern Rivers & 92.6 & 93.6 & 10.1 & 103.7 \\
\hline Mid North Coast & 98.7 & 92.7 & 10.6 & 105.5 \\
\hline Southern & 104.1 & 97.4 & 8.9 & 107.5 \\
\hline Greater Murray & 106.1 & 96.4 & 8.8 & 108.6 \\
\hline Mid Western & 111.1 & 95.9 & 8.6 & 110.8 \\
\hline New England & 115.0 & 95.7 & 7.6 & 113.5 \\
\hline Macquarie & 119.1 & 94.4 & 8.2 & 115.3 \\
\hline Far West* & 147.1 & 89.8 & 1.6 & 167.7 \\
\hline
\end{tabular}


The version of the NSW health needs index currently in use was developed in 1994, and it takes into account the influence of three factors: premature mortality (Standardised Mortality Ratio less than 65 years), socioeconomic status or EDOCC (Australian Bureau of Statistics SEIFA Index of Education-Occupation), and a rurality index (Table 1$).{ }^{6}$ The health needs index is currently under review, using data from later periods. Analysis for this review demonstrates that an additional factor should be introduced to the index: the percentage of the population that identifies as indigenous.

In 1996, additional factors were introduced to the overall RDF for the funding programs covering noninpatient services to reflect the additional needs of the indigenous people and homeless people. The rationale for introducing these factors was to provide some capacity for strategies that targeted the poor health status of these groups.

Major cost factors that are taken into account include: the extent to which private sector services meet the local population's needs; the additional costs of delivering services to dispersed rural or remote populations; the cost of interpreter services for non-English speakers; the impact of the role that principal referral hospitals play in terms of managing more severely-ill patients; teaching and research; and the effect of certain statewide services. The RDF also adjusts for the flows of patients between AHSs.

The output of the formula is a target share of resources for each AHS. Based on population projections, target shares can be developed for future years, and these targets have been used to guide the allocation of new funds across AHSs.

\section{PROGRESS IN ACHIEVING EQUITY IN RESOURCE DISTRIBUTION}

Various reports in NSW from the late 1980s noted the '...unacceptable disparities in the allocation of health resources in New South Wales', ${ }^{7}$ largely arising from the unresponsiveness of historical funding to changing population trends and health needs. ${ }^{8} 9$ Similar findings had been found earlier in the United Kingdom, when in 1974 a deliberate strategy was adopted to reduce disparities '....in terms of the opportunity for access to health care of people at equal risk'. This strategy influenced thinking in NSW to consider similar issues. ${ }^{10}$

Since the adoption of the RDF approach in the late 1980 s, considerable progress has been made in reducing the disparities in funding across NSW. In 1989-90, approximately 16.4 per cent of the health budget needed to be reallocated to achieve equity in funding. ${ }^{8}$ By 1994-95, this figure was reduced to 9.6 per cent, and by $1998-99$ it was 4.4 per cent. ${ }^{1}$ With three-year growth funding announced by the NSW Minister for Health in 2000, further progress is being made towards fairer funding for the AHSs that will further reduce these disparities. While all AHSs have received growth in funding, a greater share is being directed towards historically under-funded population growth AHSs such as those in greater western Sydney, the Central Coast, and the North Coast of NSW. The aim is to bring relatively under-funded AHSs to within two per cent of their RDF target share of resources.

\section{THE RDF'S ROLE IN REDUCING HEALTH INEQUALITIES}

It should be acknowledged that achieving equity in access to health services will not necessarily address the underlying causes of health inequalities. There may be some indirect effects. For example an equitable distribution of government-funded services tends to ameliorate broader inequalities in the distribution of income and wealth. ${ }^{11}$ Further, the health sector can play an important role in addressing geographical inequities in the distribution of employment opportunities, which is also an important influence on income and wealth distribution.

Achieving equity of access shapes the response of the health system to health inequalities as evidenced by variations in need across the population. In this respect, the RDF plays several important roles. First, equitable access may be required to ensure that once the illnesses associated with health inequalities emerge, disadvantaged populations have comparable access to effective services.

A second mechanism is through minimising the number of patients travelling long distances for routine hospital services which should be provided locally. The RDF helps achieve this by guiding a greater share of resources to develop new services in the AHSs that have historically been relatively under-serviced or have experienced rapid population growth.

In parallel with the RDF, the NSW Department of Health is implementing a system of budget holding, which will provide incentives and capacity for the AHSs to identify historical patient flows to hospitals that could be reversed through the build up of local services. While many patients travel out of an AHS for treatment for legitimate reasonssuch as proximity of services to AHS boundaries, or for specialist services that are only available in a few locations - a proportion of patient flows reflects historical referral patterns to established services that are a significant distance from the patient's home.

An important question is whether the RDF's objectives ought to be expanded beyond equity of access. This issue was at the centre of debates in 1996 over whether additional weightings should be introduced for indigenous and homeless people. These changes were 
justified on the basis of the need to target resources at groups with significantly poorer health status. In effect, this is a subtle shift from the objective of achieving equity in access towards the objective of achieving more equitable health outcomes for these groups. A serious argument, currently under consideration, is whether the formula should be enhanced to ensure resources for health programs targeted at intervening in the processes that lead to health inequalities are appropriately distributed across AHSs, in order to reflect the underlying target groups for these programs. This development may only make marginal change to the target share for each AHS, but it may embrace a more important message.

\section{LIMITATIONS}

It is important to be clear that the RDF is only one policy lever for addressing the equity issue, and by itself is an insufficient mechanism. While the RDF aims to create the broad resource capacity for equity to be achieved within the health system, an essential ingredient in delivering on equity objectives is action at the local level within AHSs. These actions may be shaped by state-level policies, but ultimately local-level strategies for addressing unmet need, and targeting of populations with relative health disadvantages, are what matter. In this context, tools for local-level decision making and resource allocation are very important.

The RDF is deliberately neutral on the issue of efficiency, and achievement of equity objectives might be frustrated by inefficient services. Other policy mechanisms are used in NSW to deal with the efficiency objective, including episode funding and hospital-cost benchmarking.

Finally, the NSW public sector health system is only part of the broader health system. While some attempts are made to take account of other sectors (such as in adjustments for private hospital use) the distribution of resources under federal programs and private finance is also important to the achievement of equity.

\section{CONCLUSION}

When combined with other strategies, the RDF is a powerful tool for addressing equity objectives in NSW. The formula will continue to be refined so that AHSs with unique factors that adversely affect the health status of their populations receive funding to improve access and meet the health needs of the population. It is also important to improve our understanding of relative differences in health need at a more micro-level, and to assist area-level decision making by refining the model to identify needs at the smaller geographic level within AHSs. A question for the immediate future is whether to broaden the objectives for the formula to include achievement of equitable health outcomes.

\section{REFERENCES}

1. NSW Department of Health. Resource Distribution Formula Technical Paper 1998-99 Revision. Sydney: NSW Department of Health, 1999.

2. Peacock S and Segal L. Equity and the funding of Australian health services: prospects for weighted capitation. Melbourne: Monash University, Health Economics Unit, Centre for Health Program Evaluation, 1999.

3. Royston GDH, Hurst JW, Lister EG, Stewart PA. Modelling the use of health services by populations of small areas to inform the allocation of central resources to larger regions. Socio-Econ Planning Sci 1992 26: 169-80

4. Carr-Hill R, Sheldon TA, Smith P, Martin S, Peacock S, Hardman G. Allocating resources to health authorities: development of a method for small area analysis of use of inpatient services. BMJ 1994 309: 1046-9.

5. Smith P, Sheldon TA, Carr-Hill R, Martin S, Peacock S, Hardman G. Allocating resources to health authorities: results and policy implications of small area analysis for use of inpatient services. BMJ 1994 309: 1050-4.

6. Eckstein $\mathrm{G}$ and Gibberd R. A relative health needs index for NSW Areas and Districts. Newcastle: Health Services Research Group, University of Newcastle, 1994.

7. NSW Department of Health. A Resource Allocation Formula for the NSW Health System. Sydney: NSW Department of Health, 1990.

8. Hannaford Committee. Report of the Western Sydney Health Taskforce. Sydney: Western Sydney Health Taskforce, 1988.

9. NSW Department of Health. The Case for a Redistribution of Health Resources. Sydney: NSW Department of Health, 1988.

10. Carr-Hill R. and Sheldon T. Rationality and the use of formulae in the allocation of resources to health care. J Public Health Med 1992; 14 (2): 117-126.

11. Schofield, D. Public Expenditure on Hospitals: Measuring the Distributional Impact. NATSEM Discussion Paper no. 37. Canberra: University of Canberra, 1999. 